HUB-EP-99/30

\title{
D-brane Spectra of Nonsupersymmetric, Asymmetric Orbifolds and Nonperturbative Contributions to the Cosmological Constant
}

\author{
Boris Körs" \\ Humboldt Universität zu Berlin \\ Institut für Physik, Invalidenstr. 110, 10115 Berlin, Germany
}

\begin{abstract}
We study nonperturbative aspects of asymmetric orbifolds of type IIA, focussing on models that allow a dual perturbative heterotic description. In particular we derive the boundary states that describe the nonsupersymmetric D-branes of the untwisted sector and their zero mode spectra. These we use to demonstrate, how some special non BPS multiplets are identified under the duality map, and give some indications, how the mismatch of bosons and fermions in the perturbative heterotic spectrum is to be interpreted in terms of the nonperturbative degrees of freedom on the type IIA side.
\end{abstract}

${ }^{1}$ Email: Koers@Physik.HU-Berlin.De 


\section{Introduction}

Quite recently certain properties of asymmetric orbifolds of type II and also type I string theories have been discussed, and they were found to display several phenomenologically attractive features, among them the opportunity to have a vanishing contribution to the cosmological constant $\Lambda_{\mathrm{c}}$, at least to leading order in perturbation theory, without supersymmetry but with a degeneracy of bosons and fermions at any mass level. Such models of type II on a torus $T^{6}$ divided out by an asymmetrically and freely acting orbifold group were constructed by Kachru, Kumar and Silverstein (KKS) in [1, 2]. They argued, that the contribution of the vacuum fluctuations to $\Lambda_{\mathrm{c}}$ might in fact be vanishing at all orders in perturbation theory. Furthermore by a self-duality argument even nonperturbative contributions are believed to be absent [3]. The applicability of these dualities in nonsupersymmetric but freely acting orbifolds derives from the well known but

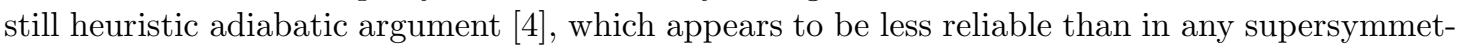
ric case.

In a slight variation of the original KKS model, two shifts exchanged and one circle decompactified but with still keeping the above mentioned perturbative features, Harvey was able to explicitly compute the nonperturbative contributions to $\Lambda_{\mathrm{c}}$ in a dual perturbative heterotic model [5]. By splitting the orbifold group in a suitable manner, one can obtain a symmetric spatial reflection in an intermediate step and employ the IIA on $\mathrm{K} 3 \times S^{1}$ to heterotic on $T^{5}$ duality. Such one obtains a dual model of the asymmetric IIA orbifold. Its partition function displayes a nonvanishing but exponentially suppressed contribution. This raises the question, if there is a way to identify the nonperturbative degrees of freedom of the IIA theory, that lead to the mismatch of bosons and fermions on the heterotic side. They should be describable as excitations of a IIA soliton compactified on $\mathrm{K} 3 \times S^{1}$ and further divided out by the asymmetric orbifold group, or of some wrapped D-brane. As we are dealing with nonsupersymmetric orbifolds, the cycles will not be holomorphic and the corresponding states cannot be deduced from any BPS argument. We shall employ the boundary state formalism to identify the non BPS D-brane spectra.

Of course, one has to worry about the question, in which situations there is any hope to extrapolate the perturbative open string analysis of strings ending on the respective brane to strong coupling, which is necessary to understand the dual spectra of the heterotic model, while the usual BPS argument is absent. A possible answer is to focus on states which are perturbatively the lightest to carry some particular conserved charge or other quantum number. These states will dominate the dynamics, if uncharged states are prohibited by the conservation law, and therefore should be present on both sides of the duality, if gauge invariance is respected. Such cases have been studied recently in a number of different dualities, including in particular dual orbifolds and orientifolds of IIB [6, 月, 8], the type I to $S O(32)$ heterotic [9] as well as the IIA on $\mathrm{K} 3$ to heterotic on $T^{4}$ dualities 10, 11]. For instance, an interpretation of a D-brane wrapping a nonsupersymmetric cycle in terms of a superposition of a brane and an anti-brane was given, that involved the appearance of tachyons in the spectra of open strings, more precisely in the Chan-Paton sector of those strings with one end on the brane and one on the anti-brane. By the application of the formerly invoked tachyon condensation [9, 12] these potentially instable states can, at least for appropriate values of the radii of the compact dimensions, have a condensate of the tachyon field on their world volume, that neutralizes their energy density everywhere except in some lower dimensional region. Such the terminology, that some tachyonic pairs of BPS branes can condensate into a single non BPS brane, defined by the vortex of the tachyon field, was established. This mechanism was justified by a strict CFT treatment for the case of a D1-brane and -anti-brane pair in type I on $\mathbb{R}^{1,8} \times S^{1}$ [9, 13], analyzing the marginal deformation that comes from the massless tachyon at a special, critical radius. It corresponds to deforming the $1+1$ dimensional CFT on the brane pair from a theory without to one with a tachyon condensate. We shall take the attitude, that this analysis can be carried over to the cases we are discussing presently without any qualitative modification. 
In the following we analyze the asymmetric orbifolds and compare some of their charged states, given by perturbative excitations of the heterotic string and of D-branes of type IIA, both subject to the orbifold projection. This allows to reveal some indication for the origin of the nonperturbative Bose Fermi mismatch of Harvey's example. The key to do this is a study of lightest charged states that can be reliably identified on both sides of the duality and also violate the degeneracy of bosons and fermions when subject to the orbifold projection. The further proceeding will be as follows: In section 2 we shall use the boundary state formalism to derive the possible BPS and non BPS Branes of IIA theory on the $\mathbb{R}^{1,9} / \mathbb{Z}_{2}=\mathrm{K} 3 \times \mathbb{R}^{1,5}$ orbifold and their zero mode spectra. In section 3 we review some aspects of the duality IIA on $\mathrm{K} 3 \times S^{1}$ to the heterotic string on $T^{5}$, in particular collect all BPS and some non BPS charges and recall, how the asymmetric elements of the orbifold group act on both sides. We shall find, that the heterotic Bose Fermi mismatch is indeed present in certain multiplets which are the lightest states to carry some charge under the gauge group. Finally in section 14 we put both together and show, how this can be recovered on a heuristic level in terms of the zero modes of D-branes on $\mathrm{K} 3 \times S^{1}$. We close with a summary.

\section{IIA on a $\mathbb{Z}_{2}$ orbifold}

We are going to construct all the possible D-brane states in the $\mathbb{Z}_{2}$ orbifold of IIA on flat spacetime, given by dividing out by a reflection $I_{4}$ in four directions, say $x_{1}, \ldots, x_{4}$. These branes carry over to the compactified theory on $\mathrm{K} 3$ at its orbifold limit $T^{4} / I_{4}$, only modified by zero mode terms. The lightest excitations of orbifold invariant superpositions of such branes will be compared to perturbative states of the heterotic asymmetric orbifold later on.

\section{$2.1 \quad$ D-brane spectra}

Examples of such states have been discussed very many times by different authors, and we only like to collect the facts we shall need, putting them into a suitably systematic order. The states will be identified using the boundary state formalism, which, to our knowledge, has been invented in [14, 15], and was used and reviewed by a couple of authors in the recent past. Such we take it as given and stick to the conventions of [7], where some of the following is to be found. For the oscillators

$|p, k, \eta\rangle=\exp \left(\sum_{n=1}^{\infty} \frac{1}{n}\left(\alpha_{-n}^{i} \tilde{\alpha}_{-n}^{i}-\alpha_{-n}^{\mu} \tilde{\alpha}_{-n}^{\mu}\right)+i \eta \sum_{r>0}^{\infty}\left(\psi_{-r}^{i} \tilde{\psi}_{-r}^{i}-\psi_{-r}^{\mu} \tilde{\psi}_{-r}^{\mu}\right)\right)|p, k, \eta\rangle_{\mathrm{NSNS}, \mathrm{RR}}^{(0)}$

solves Dirichlet boundary conditions in the $7-p i$-directions transverse to the brane and Neumann conditions in the $p+1 \mu$-directions along the brane, irrespective of the NSNS $\left(r \in \mathbb{Z}_{+}+1 / 2\right)$ or $\mathrm{RR}\left(r \in \mathbb{Z}_{+}\right)$sector groundstate $|p, k, \eta\rangle^{(0)}$. The exponential is obviously invariant under $I_{4}, \eta$ takes values \pm 1 to distinguish the different spin structures, and $k$ is the momentum eigenvalue. The use of light-cone gauge has the advantage of avoiding to write out ghost contributions, but of course it obscures covariance at several instances unavoidably. The groundstates are defined to satisfy the additional constraints coming from the zero modes. To obtain a localized state, an eigenstate of the position operator, one has to integrate over the transverse momenta $k^{\perp}$ in the Dirichlet directions:

$$
|p, \eta\rangle \equiv \delta^{(9-p)}\left(x-x^{\perp}\right)\left|p, \eta, k^{\|}=k^{\perp}=0\right\rangle=\int_{-\infty}^{\infty} d^{(9-p)} k^{\perp}\left|p, \eta, k^{\|}=0, k^{\perp}\right\rangle .
$$

One needs to introduce a relative normalization of the NSNS and RR components by 1 and $4 i$ with respect to their ground state degeneracy and an over all normalization factor $N_{\mathrm{U}}$ for the brane volume $\operatorname{Vol}_{p+1}$, which satisfies $2^{5} N_{\mathrm{U}}^{2}=\operatorname{Vol}_{p+1} /(2 \pi)^{p+1}$. The GSO invariant untwisted boundary state of type IIA is

$$
\begin{aligned}
|D p\rangle_{\mathrm{bulk}} & \equiv|D p\rangle^{\mathrm{U}} \equiv|U p\rangle_{\mathrm{NSNS}} \pm|U p\rangle_{\mathrm{RR}} \\
|U p\rangle_{\mathrm{NSNS}} & \equiv|p,+\rangle_{\mathrm{NSNS}}^{\mathrm{U}}-|p,-\rangle_{\mathrm{NSNS}}^{\mathrm{U}}, \quad|U p\rangle_{\mathrm{RR}} \equiv|p,+\rangle_{\mathrm{RR}}^{\mathrm{U}}+|p,-\rangle_{\mathrm{RR}}^{\mathrm{U}},
\end{aligned}
$$


$p$ being constrained to be even, of course. These are the well known BPS D-branes of IIA and all survive the orbifold projection. In fact only the RR component is constrained to have even $p$, while the NSNS might be of any dimension, which technically results from the fact that only the RR fields have zero modes. These are the bulk branes as opposed to the fractional ones localized at the singular fixed planes of the orbifold. The normalization convention is defined in a way, that the state is normalized to one, but carries the double amount of RR charge compared to a fractional boundary state which we turn to next.

The states of the twisted sector are necessarily localized at the fixed plane, i.e. their boundary states have to be integrated only over those momenta that are parallel to the fixed plane and transverse to the brane. The moding of the oscillators is changed from half-integer to integer and vice versa in the directions that are reflected. This leads to four (light-cone gauge) fermionic zero modes in each sector, the NSNS and the RR, giving two independent constraints on the allowed dimensions of the branes, which derive from

$$
\begin{aligned}
(-1)^{F}|r, \eta\rangle_{\mathrm{RR}}^{\mathrm{T}} & =|r,-\eta\rangle_{\mathrm{RR}}^{\mathrm{T}}, \quad(-1)^{\tilde{F}}|r, \eta\rangle_{\mathrm{RR}}^{\mathrm{T}}=(-1)^{r+1}|r,-\eta\rangle_{\mathrm{RR}}^{\mathrm{T}}, \\
(-1)^{F}|s, \eta\rangle_{\mathrm{NSNS}}^{\mathrm{T}} & =|s,-\eta\rangle_{\mathrm{NSNS}}^{\mathrm{T}}, \quad(-1)^{\tilde{F}}|s, \eta\rangle_{\mathrm{NSNS}}^{\mathrm{T}}=(-1)^{s}|s,-\eta\rangle_{\mathrm{NSNS}}^{\mathrm{T}} .
\end{aligned}
$$

Here $r+1$ is the total dimension of the brane in the orbifold fixed plane, $s$ the dimension transverse to it and $p+1=r+s+1$. In (2) one then has to replace $9-p$ by $5-r$ in the integral and the relative prefactors 1 and $4 i$ for the degeneracy of the groundstate, the dimension of the spinor, become 2 and $2 i$. Also the overall factor needs to be changed in accord with $2^{3} N_{\mathrm{T}}^{2}=4 \operatorname{Vol}_{r+1} /(2 \pi)^{r+1}$ to match the open string spectrum. The GSO invariant twisted sector boundary states are:

$$
\begin{aligned}
|D(r, s)\rangle^{\mathrm{T}} & \equiv|T s\rangle_{\mathrm{NSNS}} \pm|T r\rangle_{\mathrm{RR}} \\
|T s\rangle_{\mathrm{NSNS}} & \equiv|s,+\rangle_{\mathrm{NSNS}}^{\mathrm{T}}+|s,-\rangle_{\mathrm{NSNS}}^{\mathrm{T}}, \quad|T r\rangle_{\mathrm{RR}} \equiv|r,+\rangle_{\mathrm{RR}}^{\mathrm{T}}+|r,-\rangle_{\mathrm{RR}}^{\mathrm{T}}
\end{aligned}
$$

with even $r$ and $s$ in IIA. The most general state in the D-brane Hilbert space has twisted and untwisted components. We only consider states that carry some RR charge, so there may be long

$$
|D(p, r, s)\rangle_{\mathrm{long}} \equiv \frac{1}{2}\left(|U p\rangle_{\mathrm{NSNS}} \pm|U p\rangle_{\mathrm{RR}} \pm|T s\rangle_{\mathrm{NSNS}} \pm|T r\rangle_{\mathrm{RR}}\right),
$$

or short

$$
|D(p, r, s)\rangle_{\text {short }} \equiv \frac{1}{\sqrt{2}}\left(|U p\rangle_{\mathrm{NSNS}} \pm|T r\rangle_{\mathrm{RR}}\right)
$$

or entirely untwisted bulk branes as in (3). In particular, there are no states with odd $r$, which is of some importance for the spectrum of charges in the IIA orbifold on $\mathrm{K} 3 \times S^{1}$. This analysis is nothing but a more systematic, slight generalization of particular examples studied previously, and all the boundary states of [7, 8, 11] fit into this pattern.

\subsection{Zero mode spectra and tachyonic instabilities of non BPS branes}

We also like to collect some facts about the spectra of open strings that end on and stretch between the various D-branes [7, 8]. Their massless excitations govern the low energy dynamics and upon quantization on the worldsheet of the dual string are to be compared to the perturbative spectrum of the heterotic string. Two important issues are the amount of supersymmetry broken by the brane, to be read off from the number of its fermionic zero modes, and the appearance of tachyons in the spectrum. This is done by computing the projector $P$ that has to be introduced into the open string trace to reproduce the closed string diagram:

$$
\int_{0}^{\infty} d l\left\langle D p\left|e^{-l H_{\mathrm{cl}}}\right| D p\right\rangle=\int_{0}^{\infty} \frac{d t}{2 t} \operatorname{Tr}_{\mathrm{NS}-\mathrm{R}}\left(P e^{-2 t H_{\mathrm{Op}}}\right)
$$


Starting with the bulk brane from (3) we get the usual supersymmetric GSO projection, but no orbifold projection:

$$
P=\frac{1}{2}\left(1+(-1)^{F}\right)
$$

This state has eight (light-cone gauge, as always) fermionic and bosonic zero modes, double as many fermionic zero modes, as one would expect for a BPS state of IIA on K3, which can be resolved by observing, that only half of the states are invariant under the reflection $I_{4}$. They are the Goldstones of the broken translation invariance and the Goldstinos of the broken supersymmetries which have not yet been broken by the K3.

Only for a boundary state $|D p\rangle=|D(p, r, s)\rangle_{\text {long }}$ one finds the orbifold and GSO projection expected for an invariant fractional BPS brane:

$$
P=\frac{1}{4}\left(1+(-1)^{F}\right)\left(1+I_{4}\right)
$$

with all ambiguities concerning the relative signs of the components cancelling. The open string massless spectrum contains four bosonic and fermionic zero modes each, signalling a BPS state of the $\mathcal{N}=(1,1)$ supersymmetry in $d=6$. For a state $|D p\rangle=|D(p, r, s)\rangle_{\text {short }}$ with odd $p$ and $s$ but even $r$ the projector reads

$$
P=\frac{1}{2}\left(1+(-1)^{F} I_{4}\right)
$$

This spectrum has no GSO projection, four bosonic and eight fermionic zero modes, displaying its non BPS nature. For a superposition of a brane with its anti-brane, defined by choosing two relative signs in a superposition of two long BPS states from (6) in a way to cancel the untwisted sector RR as well as the twisted sector NSNS charges, the projection

$$
P=\frac{1}{4}\left(1-(-1)^{F}\right)\left(1-I_{4}\right)
$$

in the Chan-Paton sector of strings stretching between the two allows for four fermionic zero modes in addition to those from the other Chan-Paton sector and breaks all the supersymmetry. The question, if any of these non BPS branes or superpositions will have a tachyon in the open string NS sector, depends on the orientation relative to the orbifold fixed plane again. If the non BPS brane or the brane anti-brane pair is lying entirely inside the orbifold fixed plane, the momenta of any open string state will be even under the reflection and the NS vacuum is projected out in (11) or (12). This relative orientation enters into the closed string amplitude only through the momentum integration. If the prefactors $2^{(9-p) / 2}$ and $2^{4} 2^{(5-r) / 2}$ for the twisted and untwisted sectors leave a relative factor of 4, i.e. $p-r=s=0$ and the brane lies entirely inside the orbifold fixed plane, there is no tachyon. On the other hand, if there is a tachyon, the brane system is unstable and the mechanism of tachyon condensation should take over [9, 12. This leads to a presumably equivalent description in terms of another non BPS brane, which is assumed to be the core of the tachyon vortex. In the cases we consider this simply means, there will be a D-brane of one dimension higher or lower, that carries identical RR charges and is stable. Because of the naive identity

$$
\frac{1}{2}\left(1+(-1)^{F} I_{4}\right)=\frac{1}{4}\left(1+(-1)^{F}\right)\left(1+I_{4}\right)+\frac{1}{4}\left(1-(-1)^{F}\right)\left(1-I_{4}\right)
$$

it appears, that the non BPS brane indeed only gives a "condensed" description of the degrees of freedom of the brane anti-brane pair, all their Chan-Paton sectors being combined into a single one. But the traces, the projectors are inserted into, are in fact performed over different Hilbert spaces, differing in the momentum quantum numbers, not the oscillators. 


\subsection{Zero mode spectra of T-duality invariant superpositions}

Later on we shall be particularly interested in D-branes of IIA invariant under the naive T-duality $(-1,1)^{4}$ of all four directions of the reflection $I_{4}$ and entirely wrapped on $\mathrm{K} 3$, and thus we look at either D04, D22' or D13 superpositions:

$$
\left|D p p^{\prime}\right\rangle \equiv \frac{1}{\sqrt{2}}\left(|D p\rangle+\left|D p^{\prime}\right\rangle\right)=\frac{1}{\sqrt{2}}\left(1+(-1,1)^{4}\right)|D p\rangle .
$$

Similar superpositions of $\mathrm{D} p$ - and $\mathrm{D}(p+4)$-branes have already been encountered in asymmetric orientifolds, where in addition to an orbifold group quite similar to that used by KKS the world sheet parity was gauged [16, 17]. Fortunately, in the orientifold models there were no contributions to massless tadpoles in the sectors twisted by any asymmetric group element, and so there was no need of twisted branes. The question how twisted brane states could be defined, if they exist at all, and whether they allow any geometrical interpretation relating them to ordinary D-branes, maybe in the fashion of fractional branes, appears to be very challenging and involved. Indeed, a first step to construct such states has been taken in 18. We shall eventually argue, that there again is no necessity to include them into our analysis of charges in the asymmetric orbifold.

The $\mathrm{D} p p^{\prime}$ states above have four directions with mixed boundary conditions imposed on the open strings, therefore vanishing zero point energies in both sectors and a zero mode spectrum consisting of a single hypermultiplet of the effective theory in $d=6$, if no supersymmetry is broken by the background. The fermions in the $\mathrm{R}$ sector are invariant under the internal $S O(4)$ and those in the NS sector transform as $\mathbf{2}_{-}$, due to the extra minus of $(-1)^{F}$ from the vacuum. Then the projection by $\left(1+I_{4}\right)$ keeps one half of the fermionic zero modes of the strings between the two branes, those of the $\mathrm{R}$ sector. The state leaves one quarter of the supersymmetry invariant and from the point of view of the effective theory in $d=6$ it breaks all supersymmetry in the orbifold background. This had to be suspected, as there are no quarter BPS multiplets in the perturbative spectrum of the dual heterotic model. The spectrum can also be derived directly from the closed string amplitude, the contribution of the entire T-duality invariant superposition of $|D p\rangle \equiv|D(p, 0, p)\rangle_{\text {long }}$ states being:

$$
\begin{aligned}
& \int_{0}^{\infty} d l\left\langle D p p^{\prime}\left|e^{-l H_{\mathrm{cl}}}\right| D p p^{\prime}\right\rangle=\int_{0}^{\infty} d l\left(\left\langle D p\left|e^{-l H_{\mathrm{cl}}}\right| D p\right\rangle+\left\langle D p^{\prime}\left|e^{-l H_{\mathrm{cl}}}\right| D p\right\rangle\right), \\
& \int_{0}^{\infty} d l\left\langle D p^{\prime}\left|e^{-l H_{\mathrm{cl}}}\right| D p\right\rangle=\int_{0}^{\infty} \frac{d t}{(2 t)^{3 / 2}} 2^{3}\left(\frac{f_{3}^{4}\left(e^{-\pi t}\right) f_{2}^{4}\left(e^{-\pi t}\right)-f_{2}^{4}\left(e^{-\pi t}\right) f_{3}^{4}\left(e^{-\pi t}\right)}{f_{1}^{4}\left(e^{-\pi t}\right) f_{4}^{4}\left(e^{-\pi t}\right)}\right. \\
& \left.-\frac{f_{2}^{4}\left(e^{-\pi t}\right) f_{4}^{4}\left(e^{-\pi t}\right)+f_{4}^{4}\left(e^{-\pi t}\right) f_{2}^{4}\left(e^{-\pi t}\right)}{f_{3}^{4}\left(e^{-\pi t}\right) f_{1}^{4}\left(e^{-\pi t}\right)}\right) \\
& =\int_{0}^{\infty} \frac{d t}{2 t} \operatorname{Tr}_{\mathrm{NS}-\mathrm{R}}^{p p^{\prime}}\left(\frac{1}{4}\left(1+(-1)^{F}\right)\left(1+I_{4}\right) e^{-2 t H_{\mathrm{op}}}\right) \text {, }
\end{aligned}
$$

the trace being performed only over the strings stretching between the two branes. The $f_{i}$ were defined in [15] originally, but we use again the convention of [7], which differs by a single factor of $\sqrt{2}$ in $f_{2}$. The normalization now has to be chosen with prefactors 2 and $2 i$ for the NSNS and RR components and equally for the untwisted and twisted components: $2^{3} N_{\mathrm{U}}^{2}=$ $2^{3} N_{\mathrm{T}}^{2}=\operatorname{Vol}_{0+1} /(2 \pi)$. The state $\left|D p p^{\prime}\right\rangle$ may be $|D 04\rangle$ or $\left|D 22^{\prime}\right\rangle$. The nonvanishing terms in the numerators originate from (NS-R), $\mathrm{R} I_{4}, \mathrm{NS}(-1)^{F} I_{4}$ in the open string trace, and obviously the NS zero modes cancel. The analogous amplitude for the non BPS states $|D p\rangle \equiv|D(p, 0, p)\rangle_{\text {short }}$, e.g. $|D 13\rangle$ gives:

$$
\begin{aligned}
\int_{0}^{\infty} d l\left\langle D p^{\prime}\right| e^{-l H_{\mathrm{cl}}|D p\rangle=} & \int_{0}^{\infty} \frac{d t}{(2 t)^{3 / 2}} 2^{3}\left(\frac{f_{3}^{4}\left(e^{-\pi t}\right) f_{2}^{4}\left(e^{-\pi t}\right)-f_{2}^{4}\left(e^{-\pi t}\right) f_{3}^{4}\left(e^{-\pi t}\right)}{f_{1}^{4}\left(e^{-\pi t}\right) f_{4}^{4}\left(e^{-\pi t}\right)}\right. \\
& \left.-\frac{f_{4}^{4}\left(e^{-\pi t}\right) f_{2}^{4}\left(e^{-\pi t}\right)}{f_{1}^{4}\left(e^{-\pi t}\right) f_{3}^{4}\left(e^{-\pi t}\right)}\right)
\end{aligned}
$$




$$
=\int_{0}^{\infty} \frac{d t}{2 t} \operatorname{Tr}_{\mathrm{NS}-\mathrm{R}}^{p p^{\prime}}\left(\frac{1}{2}\left(1+(-1)^{F} I_{4}\right) e^{-2 t H_{\mathrm{op}}}\right),
$$

where only the $\mathrm{R} I_{4}$ term is missing, as compared to (15), and there are no tachyons, whatever relative signs or orientations are chosen.

\section{$3 \quad$ IIA on $\mathrm{K} 3 \times S^{1}$ and the heterotic string on $T^{5}$}

In this section we like to review the spectra of states carrying particular charges on both sides of the duality IIA on $\mathrm{K} 3 \times S^{1}$ to heterotic on $T^{5}$. The lightest among them are of special interest, as they are believed to be protected from quantum corrections in the fashion of BPS states in supersymmetric theories.

\subsection{Mapping of charges}

The way we like to figure the duality being considered first in 19, 20 relies very much on the results of 21]. We shall take the special point in the K3 moduli space, where it can be written as an orbifold $T^{4} / I^{4}$ of the torus $T^{4}$ at self dual radii and also take the compactification torus of the heterotic side to be self dual, only then the asymmetric orbifold group is a symmetry of the theory. The duality map implies 20]

$$
g_{\mathrm{II}}=\frac{1}{g_{\mathrm{het}}}, \quad r_{\mathrm{II}}=\frac{r_{\mathrm{het}}}{g_{\mathrm{het}}} .
$$

In particular it involves the essential inversion of the coupling, which is not present in the fourdimensional version of the duality. The explicit matching of the degrees of freedom is achieved by a comparison of the zero modes of the IIA solitonic ("symmetric") 5-brane [22], compactified on the K3 down to $1+1$ dimension, and the world sheet fields of the weakly coupled heterotic string on the $T^{4}$. Both consist in eight chiral, say right moving, fermions, four bosonic fields for the translation zero modes in the noncompact directions and the internal bosons on a lattice $\Gamma_{4,20}$, stemming from the compactification torus respectively from the cohomology lattice. Their classical action is derived by dimensional reduction of the supergravity action in the soliton background and coincides with the classical world sheet action of the heterotic string [21]. Of course, apparently nothing is known about how to quantize the 5-brane. This twodimensional CFT may then be compactified further to $d=5$ by putting some of the fields on an additional circle. In this spirit we shall later on apply the decomposition $\left(\boldsymbol{8}_{ \pm}, \pm\right) \rightarrow\left(\mathbf{2}_{ \pm}, \mathbf{2}_{ \pm}, \pm\right)$according to $S O(9,1) \rightarrow S O(4) \times S O(4) \times S O(1,1)$ to the fermionic zero modes. The first (broken) $S O(4)$ acts on the K3, the second on $S^{1} \times \mathbb{R}^{3} \sim \mathbb{R}^{4}$ and the unbroken $S O(1,1)$ on the world sheet $\mathbb{R}^{1,1}$ of the dual string. The chirality invariant under the K3 projection is $\left(\mathbf{2}_{+}, \ldots\right)$ and the chirality of the heterotic string world sheet fermions, respectively the Goldstone fields of the supercharges, whose symmetries are broken by the soliton, is $(\ldots,+)$.

In general we take the entire lattice $\Gamma_{5,21}$ at some generic point in the moduli space, constrained as stated above. But to allow a separate identification of charges, we factorize it into

$$
\Gamma_{5,21}=\Gamma_{16} \oplus \Gamma_{4,4} \oplus \Gamma_{1,1}
$$

for the moment, which would correspond to an enhanced gauge group $S O(32) \times S O(8) \times S U(2)$. The duality can also be "derived" from a chain of S- and T-dualities, which at some point singles out one circle of the $T^{4}$, such that the winding and Kaluza-Klein (KK) momenta on it are related to the 0 - and 4-forms, while those of the other directions of the $T^{4}$ are mapped to the 2 -form charges. Recalling, that the duality mapping exchanges the field strength of the NSNS 2-form with its dual and the vectors from the dimensionally reduced RR forms with the heterotic gauge fields form the internal lattice, we can easily write down the charges of the generic gauge group $S O(2)^{26}=U(1)^{26}$. Also we ignore, that the 3 -form potential that enters the supegravity action 
is a combination of the RR forms and the NSNS 2-form. The heterotic momenta on the $\Gamma_{16}$ are then mapped to the sixteen D0-branes located at the fixed points, or in the blow up to the D2branes wrapping the 2-cycles. At a generic point in moduli space these are not intersecting, and we shall have to turn on appropriate Wilson lines on the heterotic $T^{4}$ to break the gauge group accordingly. KK momenta and winding modes on the $T^{4}$ are related to D2-branes wrapping any of the six 2-cycles of the original torus of the K3, as well as to the D0- and D4-brane, the latter wrapping all of the K3, which together gives the $\Gamma_{4,4}$. Finally the winding and momenta of the heterotic string on the $S^{1}$ belong to the NS-5-brane of IIA wrapped entirely on $\mathrm{K} 3 \times S^{1}$. In the following section we shall see, how this simple mapping gets refined, when deforming to a generic point in the moduli space. The magnetic charges on the heterotic side are all given as some excitation of the heterotic 5-brane, leaving just one extended spatial dimension after wrapping on four of the directions of the $T^{5}$. These are mapped to perturbative KK momenta and winding excitations of the elementary IIA string, when the fifth circle of the heterotic torus remained unwrapped, and to some D-branes, when any other circle is unwrapped. Thereby the magnetic dual of any $\mathrm{D} p$-brane is a $\mathrm{D}(p-6)$-brane, as usual, wrapped in order to leave one noncompact direction. While generically the radius $r_{\mathrm{II}}$ of the fifth circle on the type II side grows large in the limit of small $g_{\text {het }}$, we could still consider any value by tuning the heterotic radius $r_{\text {het }}$, and anyway the only BPS brane wrapping this circle is the NS-5-brane, whose mass

by $r_{\mathrm{II}} / g_{\mathrm{II}}^{2} \rightarrow r_{\text {het }} g_{\text {het }}$ stays small, whereas any D-brane would acquire at least finite mass of the order of the compactification scale.

\subsection{Spectra of BPS and non BPS charged states}

In the previous paragraph we have displayed all the charges in the two supersymmetric models, now we proceed by finding the multiplets of the lightest heterotic states that carry them at the generic point of the moduli space. It is pointed out, that a given D-brane of IIA corresponds to a combination of such charges on the heterotic side. We have seen in section 2 , that there are no boundary states of the $I_{4}$ orbifold of IIA on flat space, that contained a twisted RR component and were extended in an odd number of dimensions inside the orbifold fixed plane. This continues to be valid when taking the compact version of this, IIA on a $\mathrm{K} 3 \times S^{1}$, i.e. there are no RR charged BPS or non BPS boundary states for branes of finite volume, wrapping the $S^{1}$. At the orbifold limit of the K3 the boundary states defined in section 2 are to be modified with the only respect that the momentum integration over the momenta on the $S^{1}$ direction has to be replaced by a sum over all KK momenta, and that the strings may carry winding on those directions of the $T^{4}$ that are parallel to the brane, too. Such states have been discussed e.g. in [17, 13], and the whole modification will be of no concern to us, as only the zero winding terms contribute to the massless spectrum, as long as we keep the $S^{1}$ at some generic radius at least. So we only need to regard D-branes on the K3, and these we have already examined in section 2 . Their mapping to the heterotic states charged under the appropriate gauge fields has been explored in [11], which we like to summarize briefly. For an original reference on toroidal compactifications of the heterotic string see [23].

In the perturbative heterotic spectrum precisely all states that have $N_{R}=c_{R}$, with $c_{R}$ denoting the zero point energies in the respective sectors, are BPS multiplets. On the right moving part they are vector supermultiplets of $2^{4}=16$ states. The lightest states carrying any momentum $V^{2}=2$ on the internal $\Gamma_{16}$ transform in this multiplet, while a BPS multiplet carrying only KK momentum on the $\Gamma_{4,4}$ necessarily starts with a copy of the entire massles multiplet. The Wilson lines that break the gauge group introduce a mixing of charges, as the winding states automatically have $V^{2}>0$. The charges of IIA D-branes are translated as follows: The bulk BPS D0-brane refers to heterotic states carrying KK momentum only on the special direction of the $T^{4}$, that was related to the D0- and D4-brane part of the cohomology lattice. The fractional BPS D0-brane is also charged under one of the twisted sector gauge fields in addition to the KK momentum of the bulk D0-brane. The BPS D4-branes are mapped to the winding modes in the special direction and additionally have got a nonvanishing momentum on the $\Gamma_{16}$, too. Finally 
the BPS D2-branes belong to the winding and momenta on the remaining three directions of the torus, momentum and winding being distinguished by the criterion, if the brane wraps the special circle or not, and again they carry twisted sector charge. To summarize: The only BPS charge, whose lightest states make up a gravity multiplet plus vectors, is that of the bulk D0-brane, while all other charges appear in vector multiplets on the heterotic side.

As well an example of a non BPS multiplet has been discussed. In the right moving heterotic spectrum the lightest non BPS $\left(N_{R}=c_{R}+1\right)$ multiplet is

$$
\psi_{-3 / 2}^{\mu}|0\rangle_{\mathrm{NS}}, \psi_{-1 / 2}^{[\mu} \psi_{-1 / 2}^{\nu} \psi_{-1 / 2}^{\rho]}|0\rangle_{\mathrm{NS}}, \psi_{-1 / 2}^{\mu} \tilde{\alpha}_{-1}^{\nu}|0\rangle_{\mathrm{NS}} \oplus \psi_{-1}^{\mu}|0\rangle_{\mathrm{R}}, \tilde{\alpha}_{-1}^{\mu}|0\rangle_{\mathrm{R}}
$$

and it may have $V^{2}=4$ but no winding or KK momenta on the $T^{4}$. This can be realized on the IIA side by a superposition of a D0-brane and an anti-D0-brane at different or at the same fixed points. Taken separately, they are mapped to states with $V^{2}=2$ and momenta on the $T^{4}$, that cancel each other. It leads to the projection

$$
P=\frac{1}{8}\left(1-(-1)^{F}\right)\left(1-I_{4}\right)(1+s)
$$

in the open string spectrum of the strings stretching between the different branes, where $s$ acts as a shift of the position by one half of the radius of the torus. As the projection without the shift already removes the tachyon from a D0-brane (no extension transverse to the fixed plane), the state will be stable. An alternative description of this state as a non BPS D1-brane was given [10, 11. It stretches between the two fixed points and carries a NSNS component in the untwisted sector and two twisted sector RR components. The open string spectrum has the projection

$$
P=\frac{1}{4}\left(1+(-1)^{F} I_{4}\right)(1+s) .
$$

As the D1-brane has its spatial dimension transverse to the orbifold fixed plane, it will not have the open string NS groundstate projected out in general, the lightest potentially tachyonic state being the one with minimal allowed momentum in this direction.

\subsection{The dual pair of asymmetric orbifolds}

While the models that were considered by KKS and others were defined in $d=4$, it was necessary to decompactify at least one dimension, to be able to apply a duality from IIA to the heterotic string, which inverts the coupling. On the other hand it is crucial to have still one extra circle in addition to the $\mathrm{K} 3$, as only by the shifts in this coordinate the action of the orbifold becomes

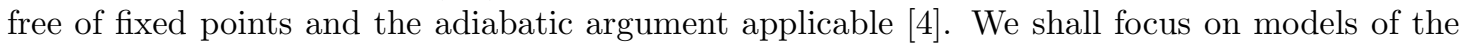
kind of that constructed by Harvey [5, where the orbifold group is generated by two elements $f$ and $g$ with $f g=I_{4}$, acting on the $T^{5}$, which we only need schematically:

$$
f \sim(-1,1)^{4}(-1)^{F_{R}} \text { and shifts, } \quad g \sim(1,-1)^{4}(-1)^{F_{L}} \text { and shifts. }
$$

It is found, that one has some freedom in arranging the shifts, which are constrained in particular by the requirement of level matching, and by $f g=I_{4}$. The orbifold projection completely eliminates supersymmetry and leaves Bose Fermi matching perturbatively, as for the KKS models, at least for the one-loop level. The group can be split off, such one can divide by $I_{4}$ first and only then by an asymmetric element, say $f$. After using the duality of IIA on $T^{4} / I_{4} \times S^{1}$ to heterotic on $T^{5}$ in the first step, one deduces the action $f_{\text {het }}$ of $f$ on the heterotic side, and as it is free, hopes for the duality to be inherited by the pair of nonsupersymmetric orbifolds:

$$
f_{\text {het }} \sim\left((-1)^{8}, 1^{8}\right)_{I}(-1,1)_{i}^{4}(-1)^{F_{R}} \text { and shifts. }
$$

There may appear to be some ambiguity in how to generalize the transformation properties under $f_{\text {het }}$ from the massless fields to the massive. On the IIA side the zero modes of the 5-brane give 
the world sheet field theory of the heterotic string after compactification on K3. Their transformation under $f$ can directly be deduced from the tendimensional massless fields, and it is just the action we have given above: One half of the RR forms get reflected and only the NSNS fields with no component in the $I_{4}$ directions are invariant. Then by duality this has to be the action on the heterotic world sheet fields without anything left to be guessed, except for the shifts.

In the twisted sector of the heterotic orbifold there are no massless fermions anyway, due to the positive zero point energy, and the only boson of potentially zero mass is the NS ground state, whose mass depends on $r_{\text {het }}$, the tachyonic region, depending on the nature of the shift on the $\Gamma_{1,1}$, at large or small radius. In particular the twisted sector does not display any new massless vector fields, such that there are no new gauge degrees of freedom and no new charges present. Correspondingly there should be no massless RR forms in the twisted sector of the dual IIA orbifold showing up and therefore no twisted sector D-branes or boundary states to be regarded. This argument is surely not entirely satisfactory and should be accompanied by an explicit construction of the twisted sector boundary states or a proof of their absence. Unfortunately we do not know a way to do this presently.

Let us also note the action of $f_{\text {het }}$ on the multiplets with $\left(N_{L}=1, N_{R}=c_{R}\right)$ and $\left(N_{L}=\right.$ $\left.0, N_{R}=c_{R}+1\right)$ explicitly. Any of the lightest state carrying a given RR charge transforms in one of these (or a BPS vector multiplet $\left(N_{L}=0, N_{R}=c_{R}\right)$ ). We assume to have chosen a combination of charges that is invariant under the action of $f_{\text {het }}$ on the lattice, so there will be no further signs to be considered. In the first case one half of the gauge degrees of freedom are invariant and of the graviton multiplet all the gravitinos and one half of the vectors are odd. This leaves Bose Fermi matching in the massless spectrum and even a supermultiplet for the diagonal of the gauge group. The second is the lightest non BPS multiplet. The projection onto invariant states simply removes all fermions and leaves all bosons. We immediately recognize the violation of Bose Fermi matching at the massive level of the untwisted sector. In particular it is manifest in non BPS multiplets that will be found to be the lightest to carry some charge under the gauge group in the orbifold.

\section{Charged states on the asymmetric orbifolds}

In the preceding sections we have collected a couple of facts about the spectra of states carrying some particular charges under the gauge group on both sides of the (conjectured) duality. On the heterotic side these are perturbative states of the untwisted sector, invariant under the orbifold projection. On the IIA side the charges map to superpositions of D-branes. Their zero modes should, after quantization on the worldsheet of the dual string, reproduce at least the lightest heterotic states of a given charge. While we do not have a precise understanding, how the supergravity solution should be modified on the asymmetric orbifold, we have been able to deduce the zero mode spectra of D-branes from the according boundary states. We shall now figure out, which space-time fields they would generate as their lightest excitation, if taken to be world sheet fields of the dual string, subject to the asymmetric orbifold projection, and compare to the spectra of non BPS states on the heterotic side.

\subsection{Fractional BPS branes}

We first look at the boundary states of the $\mathrm{K} 3 \times S^{1}$ orbifold, as derived in section 2, and afterwards we come to the invariant superpositions of branes in the untwisted sector of the asymmetric orbifold. Branes of the twisted sector do not need to be inspected, as there are no additional charges arising, due to the absence of further gauge fields. Recall first, that the BPS soliton of IIA breaks the supersymmetries

$$
\left(\mathbf{2}_{+}, \mathbf{2}_{ \pm},+\right),\left(\mathbf{2}_{-}, \mathbf{2}_{ \pm},-\right)
$$


of even chirality on its worldvolume [22], referring to the zero modes kept by the usual GSO projection of the open strings ending on a BPS bulk brane. The K3 leaves all spinors $\left(\mathbf{2}_{+}, \ldots\right)$ invariant, i.e. those that are even under $I_{4}$. The zero modes on the fractional branes that are invariant under the orbifold projection in the open string sector via (10) are then given by those which are unbroken by the $\mathrm{K} 3$ and even under $(-1)^{F}$ :

$$
\left(\mathbf{2}_{+}, \mathbf{2}_{ \pm},+\right) .
$$

They are both of positive chirality on the world sheet, identified with the right moving world sheet spinors of the heterotic theory. After the quantization of the world sheet theory of the dual string they generate a ground state degeneracy of $2^{8 / 2}=16$ and reproduce as their lowest excitation the tendimensional BPS vector multiplet, which the lightest states carrying the charge of the D-brane in the perturbative heterotic spectrum transform in 21]. Also we notice, that the heavier states of the heterotic string, carrying the same charge, would involve excitations of all left moving bosonic oscillators on the world sheet, whereas the zero mode spectrum of the D-brane involves only the bosonic Goldstone fields for the translations transverse to the K3, not enough to reproduce the heterotic multiplets.

\subsection{Non BPS branes}

The more interesting case is that of the non BPS brane with nonsupersymmetric projection (11) in the open string spectrum. In addition to the zero modes of the fractional brane from open strings with GSO projection there are those, which are odd under $(-1)^{F}$ and $I_{4}$, therefore belong to supersymmetries that remained unbroken by the BPS soliton and transform as $\left(\mathbf{2}_{-}, \ldots\right)$, together:

$$
\left(\mathbf{2}_{+}, \mathbf{2}_{ \pm},+\right),\left(\mathbf{2}_{-}, \mathbf{2}_{ \pm},+\right) .
$$

These sixteen zero modes are still chiral on the world sheet, as they better had to, and upon quantization they generate a degeneracy of $2^{16 / 2}=256$ on the right moving side, which, subject to the GSO projection in $d=10$, gives rise to 16 fermions, half of them even, the other half odd under $I_{4}$. This looks exactly like the fermionic states as given in (19) for the lightest non BPS multiplets of the heterotic string with $V^{2}=4$. Again the more massive states are not reproduced correctly by the single D-brane, as not enough bosons are available. Up to now we have omitted the tachyon that may be present in the open string spectrum on the IIA side. In fact it will be there, as in general the branes are extended on the K3. But the open string sector with momenta odd under $I_{4}$ does not have any additonal zero modes, except at the special radius, when the tachyon gets massless. The deformation that is associated with this additional bosonic field was shown, even if only in a quite different situation, to allow to pass to an equivalent theory in a lower dimension but with a tachyon condensate [9, 12]. If this mechanism works in our case, it will reduce or enlarge the number of dimensions transverse to the orbifold fixed plane, acquiring some condensate, which only neutralizes the energy density of the brane in these directions, and finally the above argument goes through without any additional zero modes to be regarded. In the present case for instance a D1-brane stretching between two fixed points gets deformed to a pair of D0-branes, brane and anti-brane, sitting at the two fixed points. The oscillator parts of the open string Hilbert spaces only differ by states, which had combinations of momenta on the D1-brane, that were odd under $I_{4}$. Therefore the zero modes of the stable brane anti-brane configuration with tachyon condensate are just those of the unstable non BPS brane and the spectra of charged states identical. In this sense (13) really gets to work after tachyon condensation. In the supersymmetric models the bosonic states should then follow automatically, indeed.

\subsection{Branes of the asymmetric orbifold}

Only little remains to do the same analysis for the states of the untwisted sector of the asymmetric orbifold. Following our discussion of section 3, it acts on the entire $\Gamma_{4,20}$ by a reflection in 
the sense, that the momenta on the $\Gamma_{16}$ are exchanged in some fashion and the winding and KK momenta on the $\Gamma_{4,4}$ also. On the type II side this amounts to mapping fractional D0-branes to D4-branes with Wilson lines given by the image of the charge of the D0-brane on the $\Gamma_{16}$. D2-branes wrapping a 2-cycle of the $\Gamma_{4,4}$ are mapped to the D2-branes wrapping the naively dual 2-cycle in the other two directions, and their twisted charges on the $\Gamma_{16}$ also get exchanged. But the obvious invariant combinations, a brane and its image, are not the lightest states to carry any given charge. Such an invariant D22'-brane superposition would carry winding and momentum on the $T^{4}$ as well as internal momentum with respect to two of the $S O(2)$ from the $\Gamma_{16}$ in the heterotic picture. But, of course, there are also heterotic states which carry invariant combinations $V^{2}=\left(v_{1}+v_{2}\right)^{2}=4$ of internal momenta, but their winding and momenta on the $T^{4}$ vanish. These are mapped to brane anti-brane superpositions, the lightest states to carry any orbifold invariant charge on the $\Gamma_{16}$. The D0-brane anti-brane pair, respectively the presumably equivalent non BPS D1-brane, has already been analyzed above as a non BPS state, which carried the fermionic zero modes to generate the space-time fermions of (19), the heterotic non BPS multiplet, and was stable for some complementary regions of the radii. The orbifold projection of IIA applied on the world sheet of the dual string now gives a minus to all fermions, that are left moving on the world sheet and odd under $I_{4}$ by $(-1,1)^{4}$, i.e. to none, and a minus to all, that are right moving by $(-1)^{F_{R}}$, i.e. to all. All the fermions are removed and only bosons left, the same action as on the heterotic non BPS multiplet, which was a source of the violation of the matching of bosons and fermions. Note, that we used the orbifold projection on the IIA side only to deduce this and did not require any information on the heterotic translation $f_{\text {het }}$. While this approach gives an interpretation of how the mismatch emerges in the nonperturbative regime of the IIA orbifold, it surely does not allow to do any quantitative computation of the contributions to the partition function.

There are several other cases left, one has to consider, for instance the superposition of a D4or D2-brane with an anti-brane wrapping the same directions of the torus. The lightest heterotic oscillator excitations are again given by (19) and the derivation of the zero modes proceeds as above.

One might think, that there also were states carrying equal winding and KK momentum quantum number on the $T^{4}$ but no twisted sector charges. In fact no such superpositions without momentum on the $\Gamma_{16}$ can occur, as the presence of Wilson lines enforces $V^{2}>0$ for winding states. The application of $f_{\text {het }}$ does not map the BPS states with KK momentum and $V^{2}=v_{1}^{2}=2$ to the BPS winding states with $V^{2}=\left(v_{2}+A\right)^{2}=2, A$ indicating the Wilson line in the direction of the winding, for instance as defined in 11]. Also one needs to notice, that the left handed reflection enforces winding equal to minus the canonical momentum, as opposed to a right handed reflection. There are in fact invariant states in the heterotic spectrum with opposite momentum and winding as well as $V^{2}=\left(v_{1}+v_{2}+A\right)^{2}=4$. The level matching demands for the lightest such states $N_{L}=0$ and $N_{R}=c_{R}$, which before orbifolding is the BPS vector multiplet. The obvious candidate for an invariant boundary state to describe the dual state with the required $\mathrm{RR}$ charges is a superposition of a D2-brane and an anti-D2-brane wrapping disjoint circles of the $\mathrm{K} 3$, according to opposite momentum and winding. It is one of the stable superpositions with mixed boundary conditions in four directions, which we have discussed in section 2, with the exception, that in the Chan-Paton sector of open strings between the two branes the projector is

$$
P=\frac{1}{4}\left(1-(-1)^{F}\right)\left(1-I_{4}\right)
$$

but again no tachyons can appear. In the other Chan-Paton sectors it has the fermionic zero modes of a fractional brane, whereas in the mixed sector all zero modes from the R and NS sectors now are projected out. The resulting zero mode spectrum is that of a fractional D0-brane, which again generates a chiral world sheet theory on the dual string with appropriate degeneracy of the vacuum to reproduce the BPS vector multiplet as its lowest excitation. The orbifold projection then removes the fermions and leaves the bosons, as before. 
The D22' superposition is presumably equivalent via tachyon condensation to an instable superposition of a D3- and a D1-brane both stretching between the two fixed points and with opposite bulk charges. In this spirit D0- anti-D4-brane superpositions and their tachyonic equivalent, a D1- anti-D3-brane superposition wrapping the special circle, can also be treated.

\section{Summary}

We have analyzed the spectra of boundary states that arise in the untwisted sector of a particular class of asymmetric orbifolds. They were found to be given by superpositions of branes of the original IIA theory on K3, that are invariant under a version of T-duality. The lightest states carrying a given conserved charge were found to consist of brane anti-brane pairs. For any such pair there was an alternative description in terms of non BPS branes, the equivalence defined via the condensation of the tachyon arising in the open string spectrum. Assuming the validity of the adiabatic argument and the continuity of some charged non BPS states, the zero modes of the open strings on these branes were shown to be chiral on the world sheet of the dual string and to be candidates to generate a spectrum of space-time fermions that have the correct multiplicity and the same transformation properties under the orbifold reflection $I_{4}$, as the lightest heterotic states that carry the same charge. This may be figured to be a consistency check of the applicability of the adiabatic argument and the underlying duality and as a suggestion, how the mismatch of bosons and fermions in the nonperturbative regime of the IIA orbifold appears.

\section{Acknowledgements}

I like to thank A. Krause, A. Miemiec and in particular R. Blumenhagen for valuable discussion, as well as M. Gaberdiel for answering a question of mine.

\section{References}

[1] S. Kachru, J. Kumar, and E. Silverstein. Vacuum Energy Cancellation in a NonSupersymmetric String. Phys. Rev. D 59 p. 106004, 1999.

[2] S. Kachru and E. Silverstein. On Vanishing Two Loop Cosmological Constants in Nonsupersymmetric Strings. JHEP 9901 p. 4, 1999.

[3] S. Kachru and E. Silverstein. Self-Dual Nonsupersymmetric Type II String Compactifications. JHEP 9811 p. 1, 1998.

[4] C. Vafa and E. Witten. Dual String Pairs with $N=1$ and $N=2$ Supersymmetry in Four Dimensions. Nucl. Phys. Proc. Suppl. 46 p. 225, 1996.

[5] J. A. Harvey. String Duality and Non-supersymmetric Strings. Phys. Rev. D 59 p. 26002, 1999.

[6] A. Sen. Stable Non-BPS States in String Theory. JHEP 9806 p. 7, 1998.

[7] A. Sen. Stable Non-BPS Bound States of BPS D-branes. JHEP 9808 p. 10, 1998.

[8] O. Bergman and M. R. Gaberdiel. Stable non-BPS D-Particles. Phys. Lett. B 441 p. 133, 1998.

[9] A. Sen. SO(32) Spinors of Type I and other Solitons on Brane Antibrane Pair. JHEP 9809 p. 23,1998 .

[10] A. Sen. BPS D-branes on Non-supersymmetric Cycles. JHEP 9812 p. 21, 1998. 
[11] O. Bergman and M. R. Gaberdiel. Non-BPS States in Heterotic-Type IIA Duality. JHEP 9903 p. 13, 1999.

[12] A. Sen. Tachyon Condensation on the Brane Anti-Brane System. JHEP 9808 p. 12, 1998.

[13] M. Frau, L. Gallot, A. Lerda, and P. Strigazzi. Stable Non-BPS D-Branes in Type I String Theory. (hep-th/9903123).

[14] C.G. Callan, C. Lovelace, C.R. Nappi, and S.A. Yost. Loop Corrections to Superstring Equations of Motion. Nucl. Phys. B 308 p. 221, 1988.

[15] J. Polchinski and Y. Cai. Consistency of Superstring Theories. Nucl. Phys. B, 1988.

[16] R. Blumenhagen and L. Görlich. Orientifolds of Non-supersymmetric, Asymmetric Orbifolds. Nucl. Phys. B 551 p. 601, 1999.

[17] C. Angelantonj, I. Antoniadis, and K. Förger. Non-Supersymmetric Type I Strings with Zero Vacuum Energy. (hep-th/9904092).

[18] I. Brunner, A. Rajaraman, and M. Rozali. D-Branes on Asymmetric Orbifolds. (hepth/9905024).

[19] C.M. Hull and P.K. Townsend. Unity of superstring dualities. Nucl. Phys. B 438 p. 109, 1995.

[20] E. Witten. String Theory Dynamics in Various Dimensions. Nucl. Phys. B 443 p. 85, 1995.

[21] J.A. Harvey and A. Strominger. The Heterotic String is a Soliton. Nucl. Phys. B 449 p. 535, Erratum Nucl. Phys. B 458 p. 456, 1995 and 1996.

[22] C.G. Callan, J.A. Harvey, and A. Strominger. Worldbrane Actions for String Solitons. Nucl. Phys. B 367 p. 60, 1991.

[23] P. Ginsparg. On Toroidal Compactifications of Heterotic Superstrings. Phys. Rev. D 35 No. 2 p. $648,1987$. 